\title{
HUMAN RESOURCE AND PECULIARITIES OF INCOME DISTRIBUTION
}

\author{
RAMAZ OTINASHVILI \\ Doctor of Economic Sciences, Professor \\ Georgian Technical University, \\ Academician of the Academy of Economic Sciences of Georgia, Georgia \\ R.Otinashvili@gtu.ge
}

Abstract. The country's leading factor in human development that is a human resource. It is the creator of everything and it is necessary to create a law and protect human resource. People need to implement, pursue the economic policy and ensure business efficiency. That functions, to prevent crime and to restore order. Man builds the country, but to build well, knowledge is needed and knowledge needs education. Humans capital is a body of knowledge and includes philosophical, social, economic and psychological aspects. This term dates from the eighty-seventh year of the twentieth century to its end. It is founder is Nobel Prize Laureate G. Becker. Human capital consists of: educational, professional-quality, information-cultural and social connections. Among them, the capital of the Social Union dominates in the conditions of Georgia. According to the GAERO, Georgia ranks 61st out of 189 countries in the Human Development Index. Human resources determine a person's income. It is important to note that human capital is one of the main causes of income inequality. We differ from each other in terms of data and capabilities. It's important that state should have a fair social-economic environment, which gives all possible conditions to citizens for the realization of opportunity.

KEYWORDS: HUMAN CAPITAL, BUSINESS, INCOME, NATURAL RESOURCE, PROPERTY, ECONOMIC POLICY.

For citation: Otinashvili, R., (2021). Human Resource and Peculiarities of Income Distribution. Globalization and Business, №11, 101-107. (In Georgian). https://doi.org/10.35945/gb.2021.11.014 


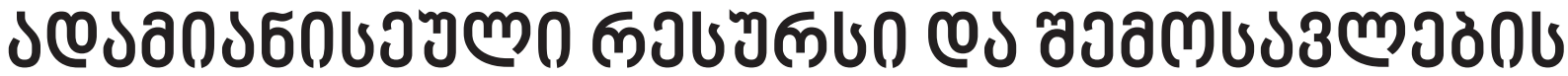

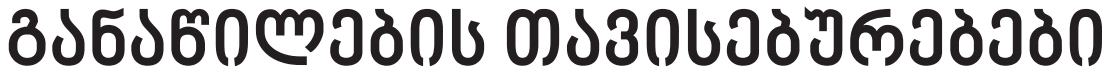

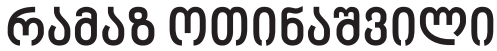

эзмбмдаз зुल

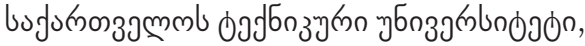

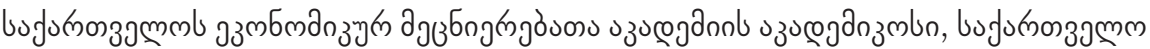

R.Otinashvili@gtu.ge

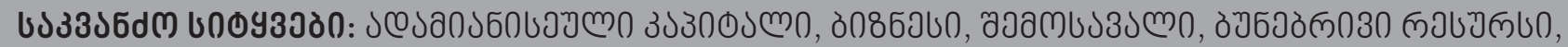

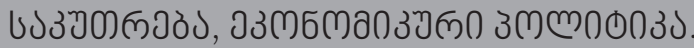

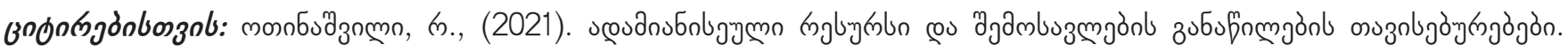

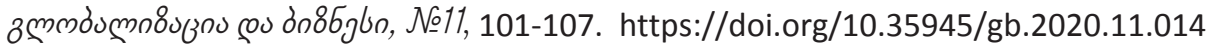

\section{әอง১ว১ल0}

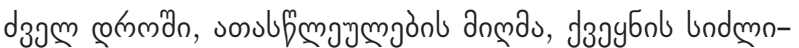

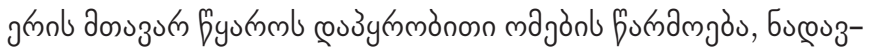

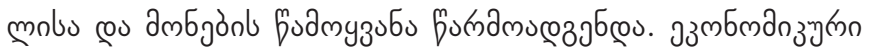

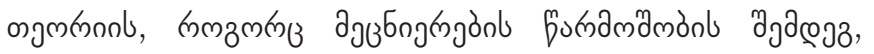

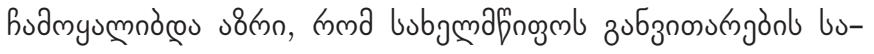

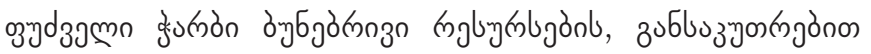

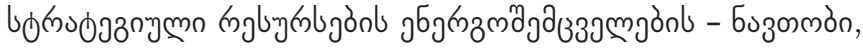

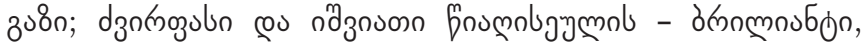

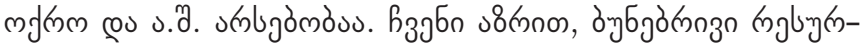

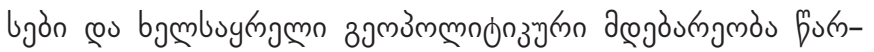

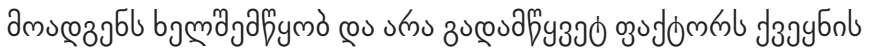

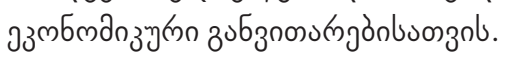

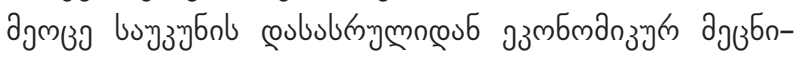

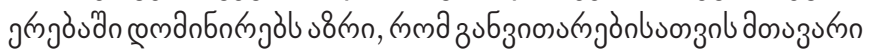

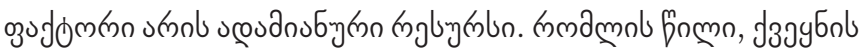

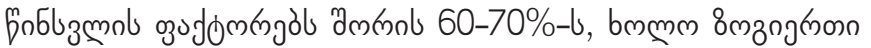

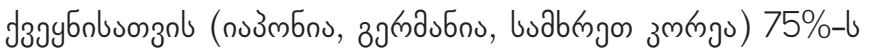

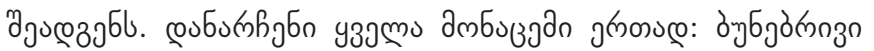

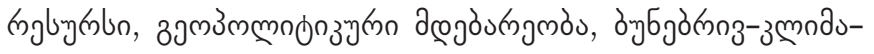

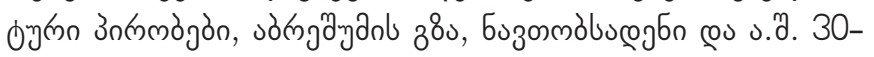

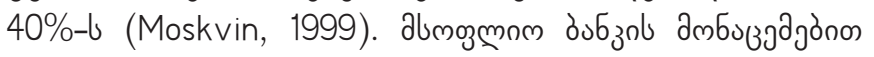

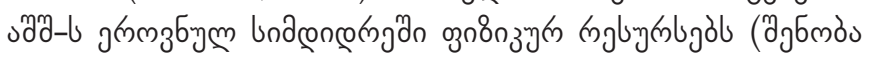

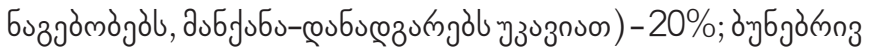

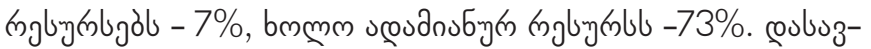

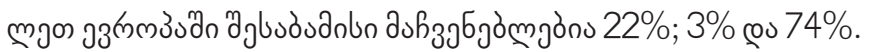

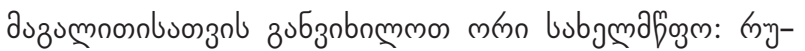

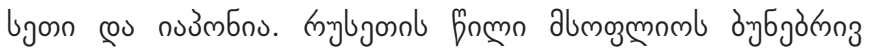

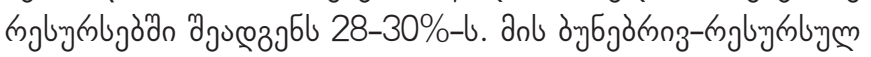

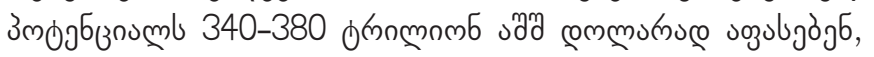

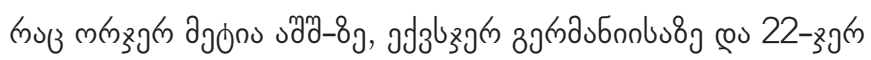

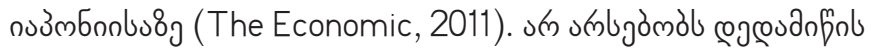

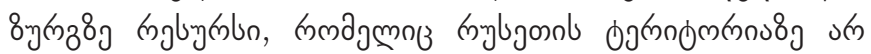

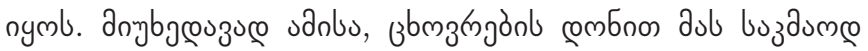

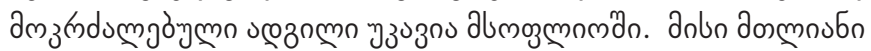

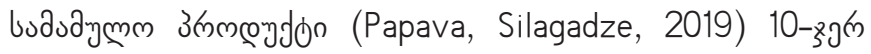

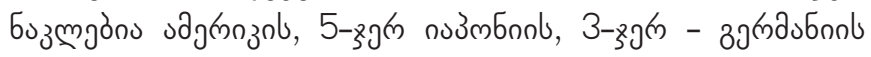

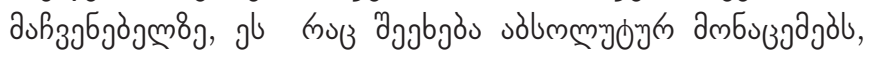

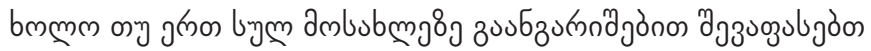

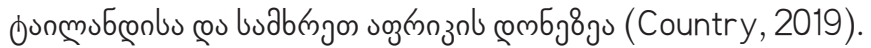

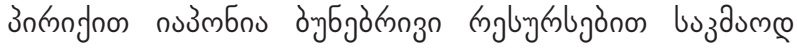

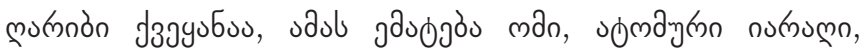

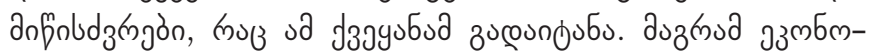

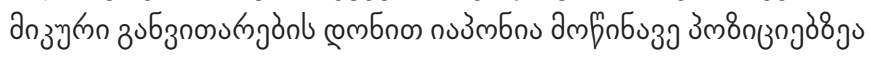
abmoुmnnḡn.

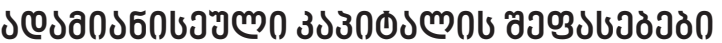

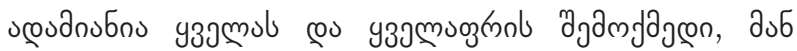

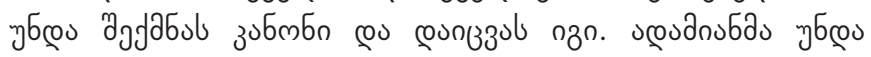

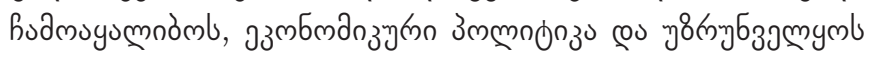

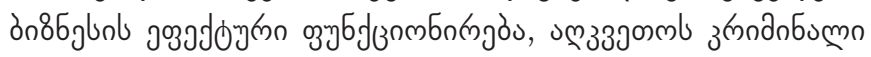

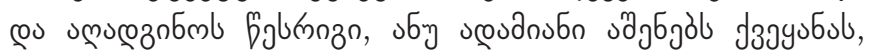

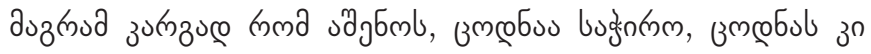

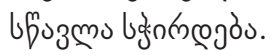

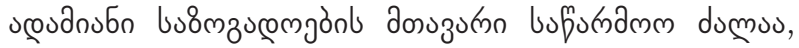

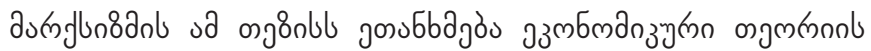

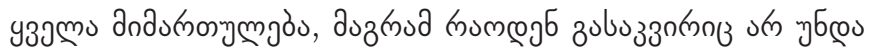

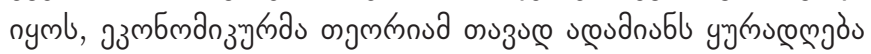

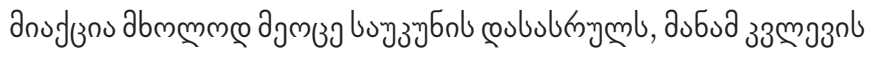




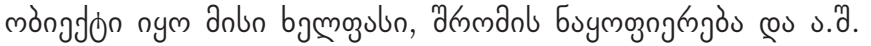
(Abralava, 2014).

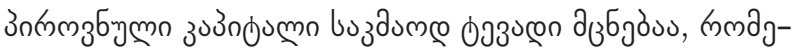

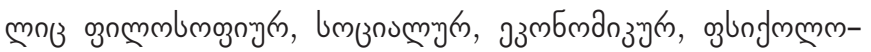

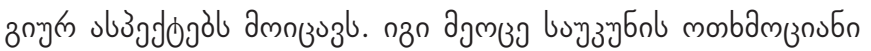

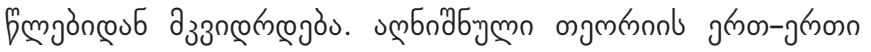

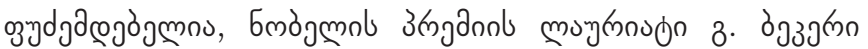

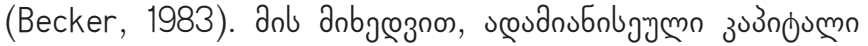

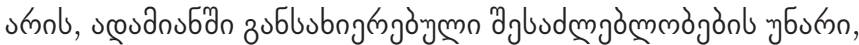

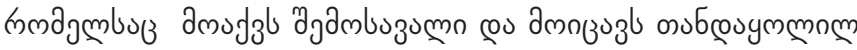

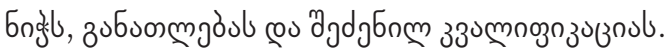

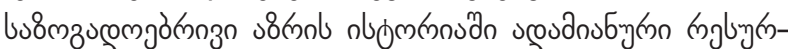

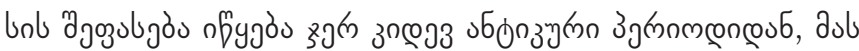

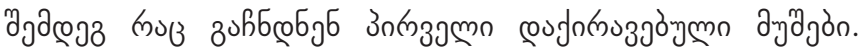

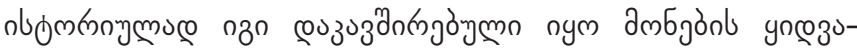

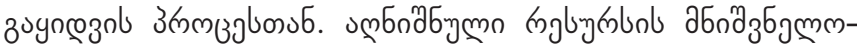

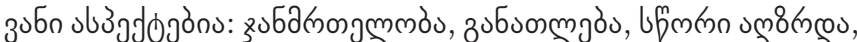

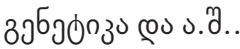

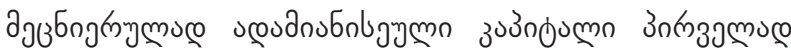

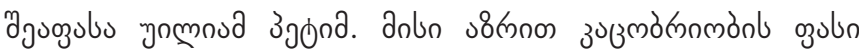

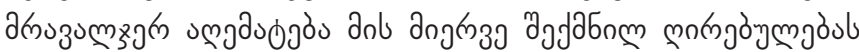
(Sartania, Tokmazishvili, Maglakelidze, 2004). дmz3ns-

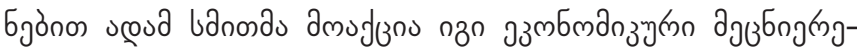

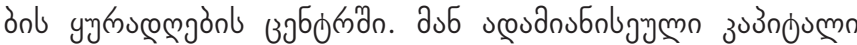

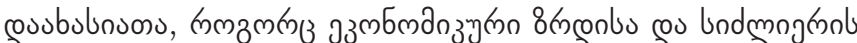

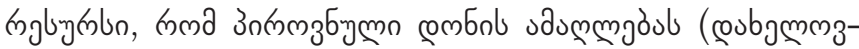

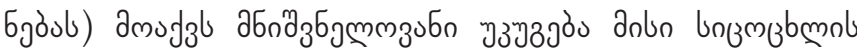
उ

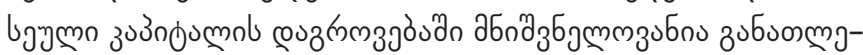

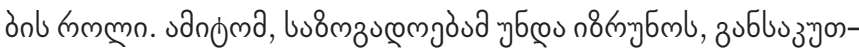

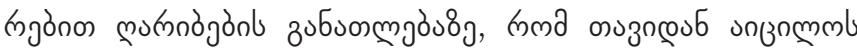

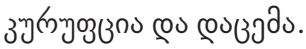

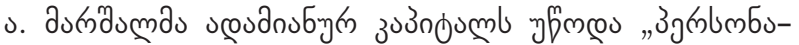

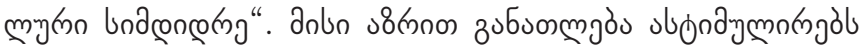

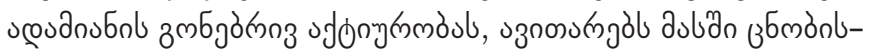

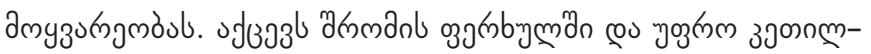

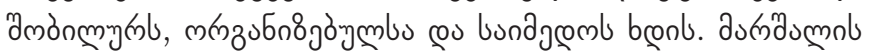

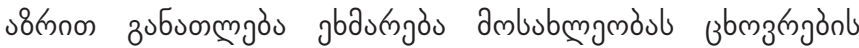

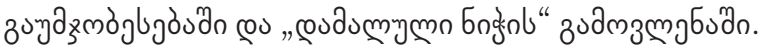

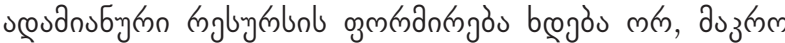

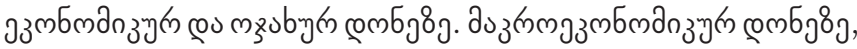

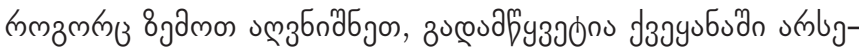

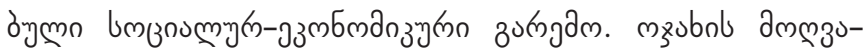

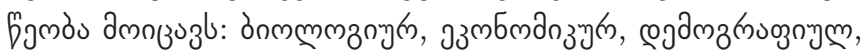

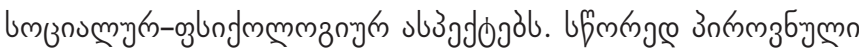

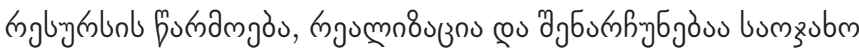

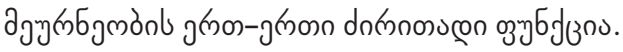

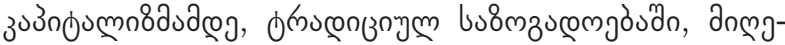

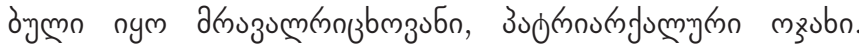

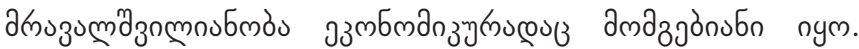

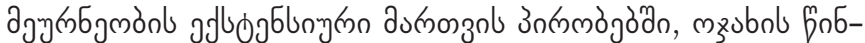

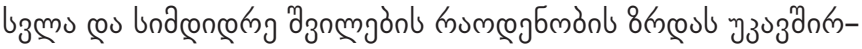

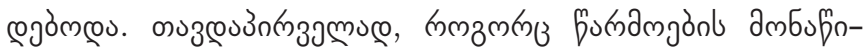

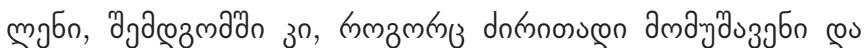

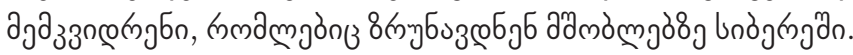

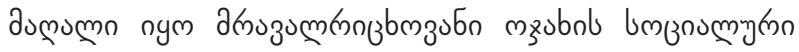

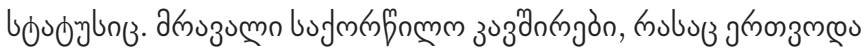

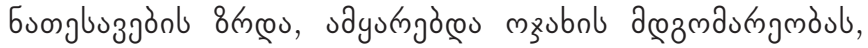

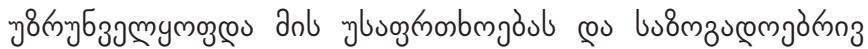
boudybl.

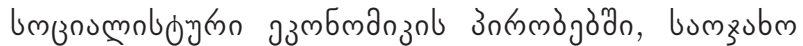

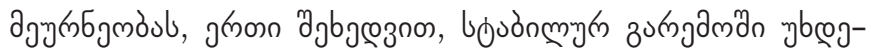

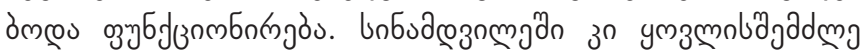

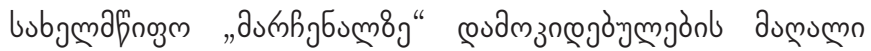

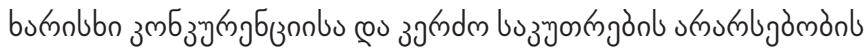

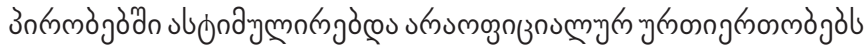
(Papava, 2018).

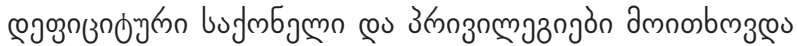

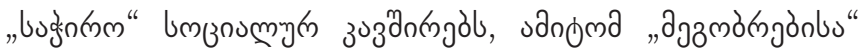

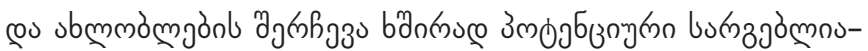

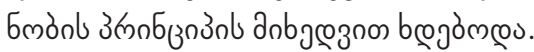

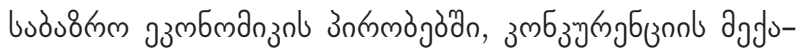

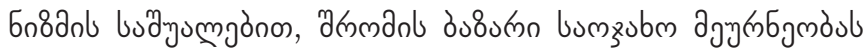

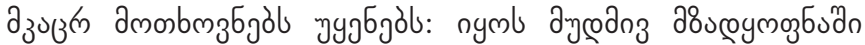

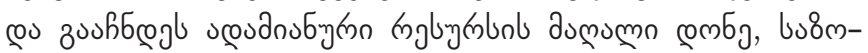

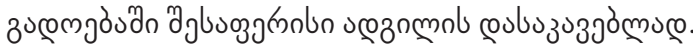

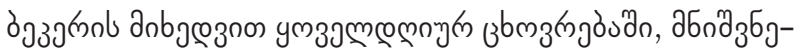

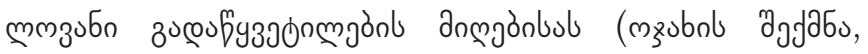

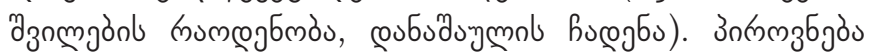

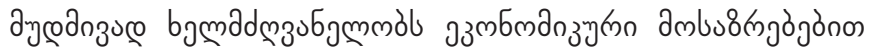

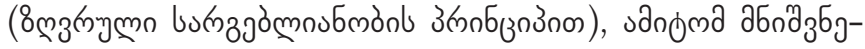

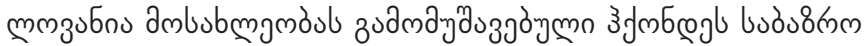
Һзว3วoेn. 2002):

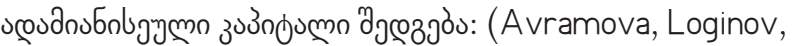

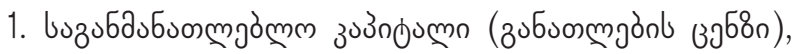

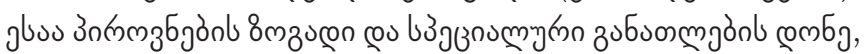

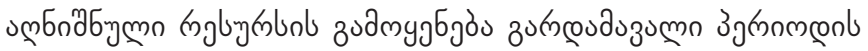

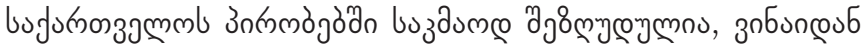

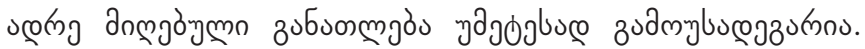

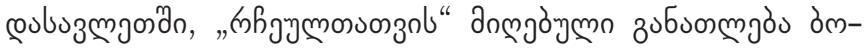

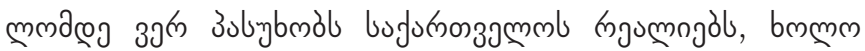

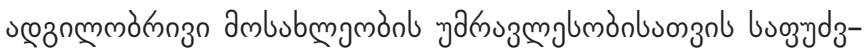

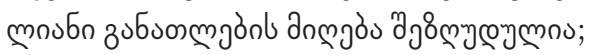

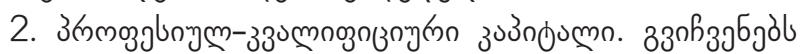

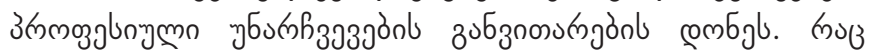

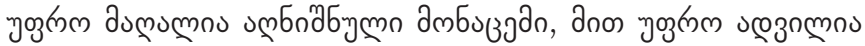

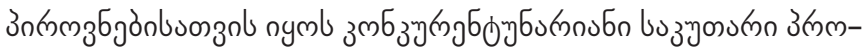

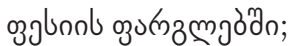

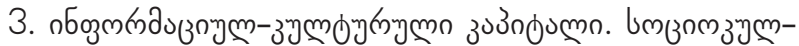




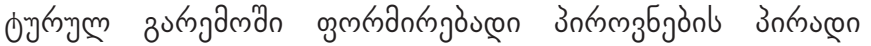

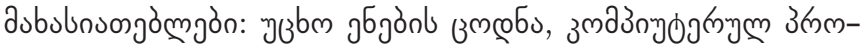

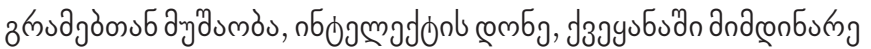

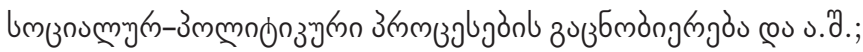

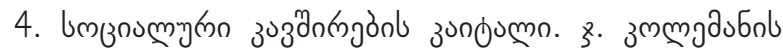

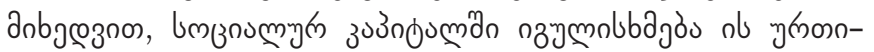

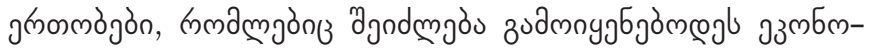

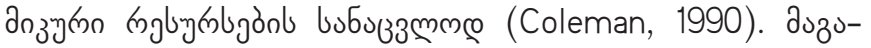

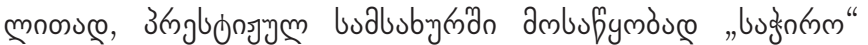
bumboll bymagafymò.

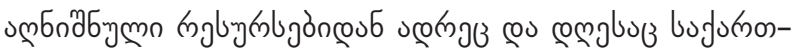

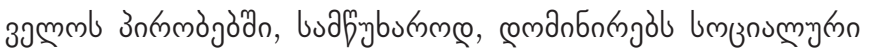
зu3an

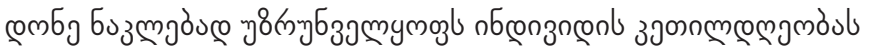

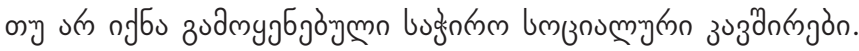

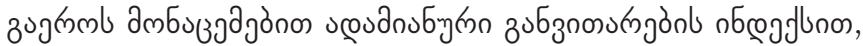

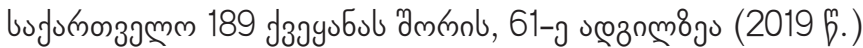
(http://hdr.undp.org/sites/default/files/hdr2020.pdf).

\section{әวамьзамо буљљпวдо}

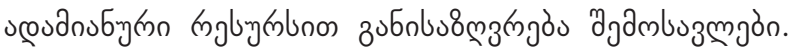

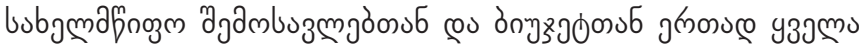

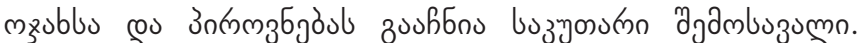

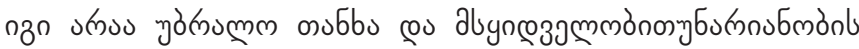

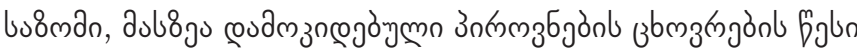

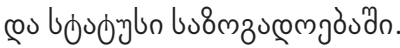

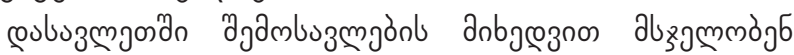

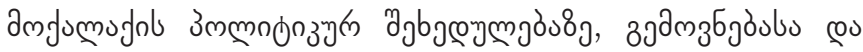

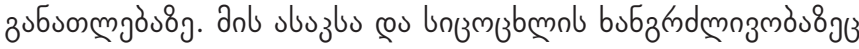

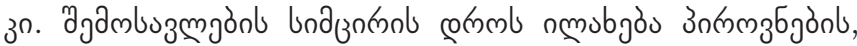

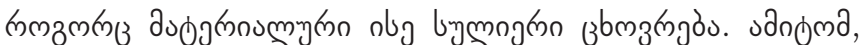

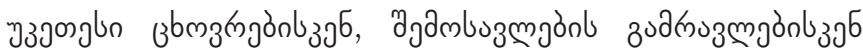

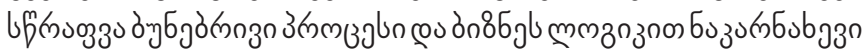

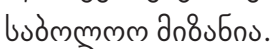

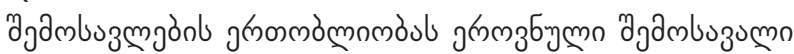

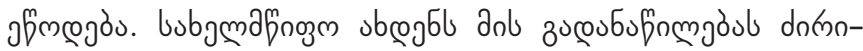

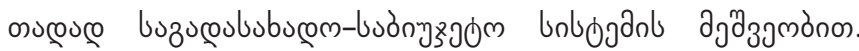

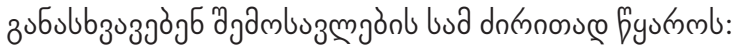

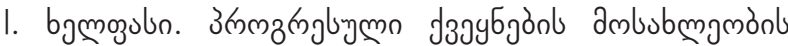

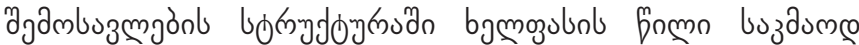

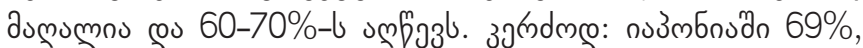

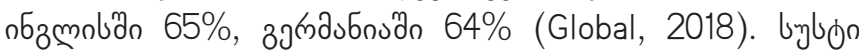

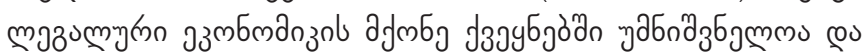

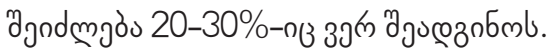

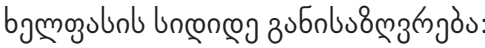

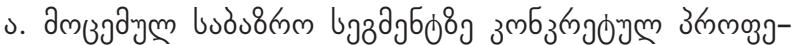

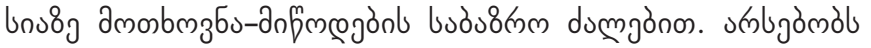

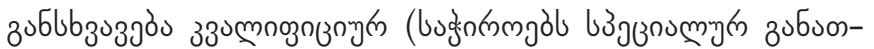

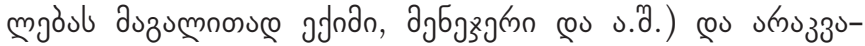

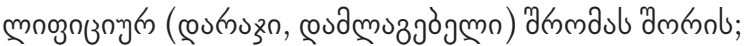

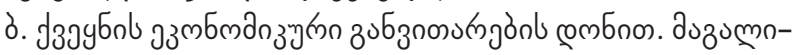

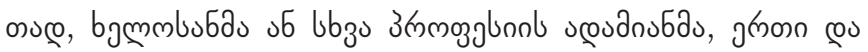

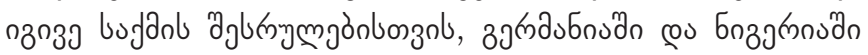

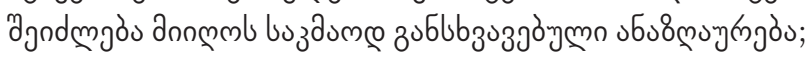

3. bymogubnt unenœ

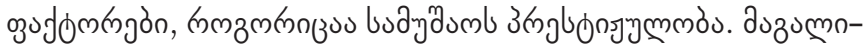

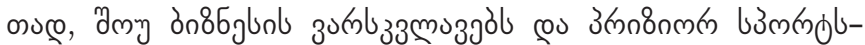

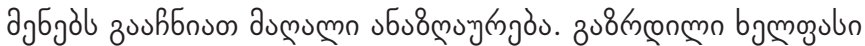

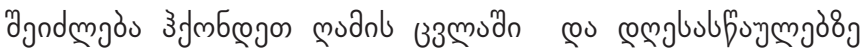

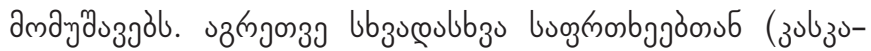

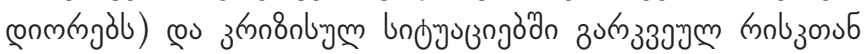

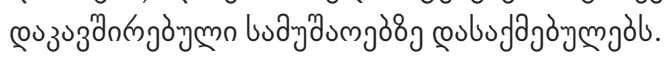

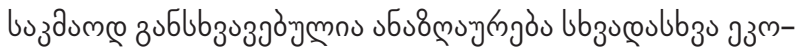

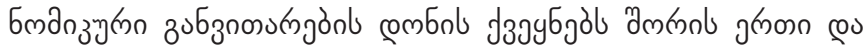

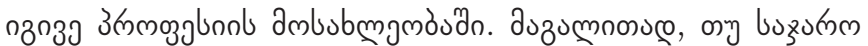

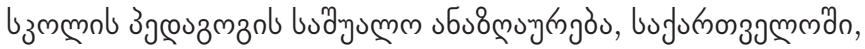

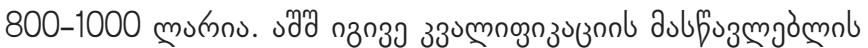

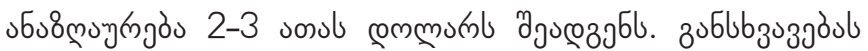

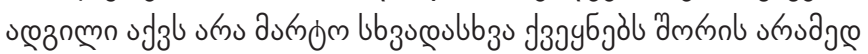

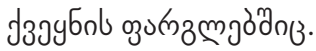

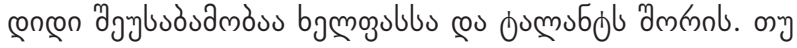

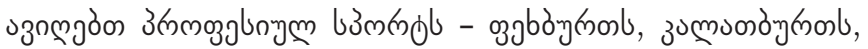

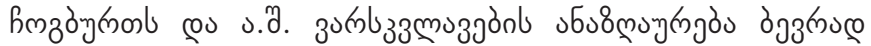

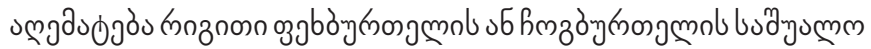

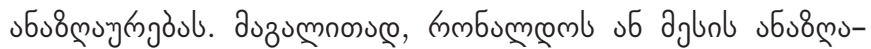
चங

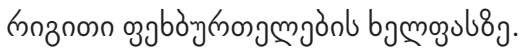

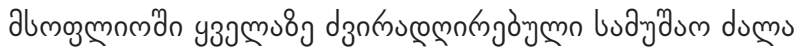

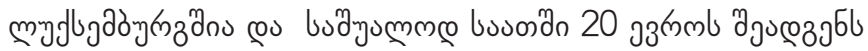

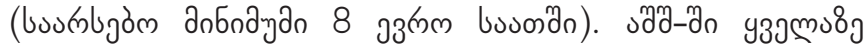

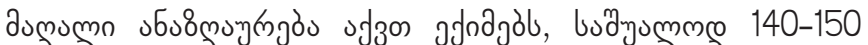

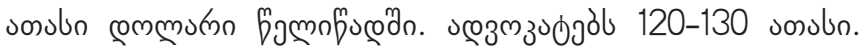

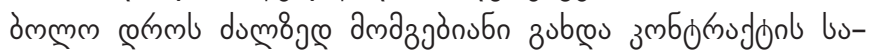

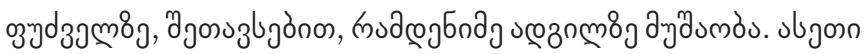

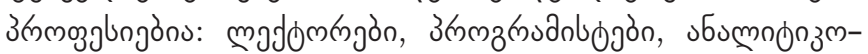

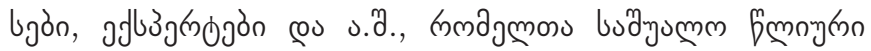

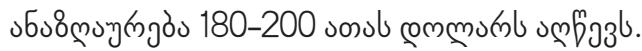

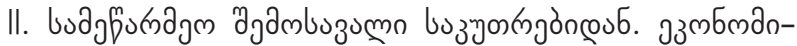

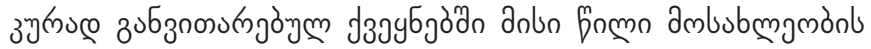

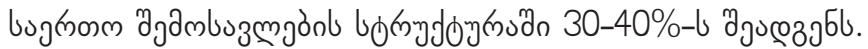

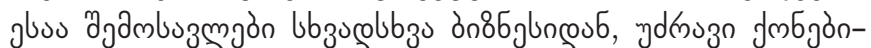

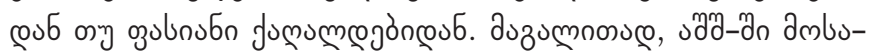

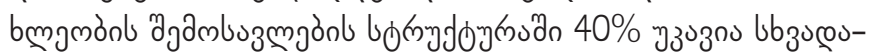

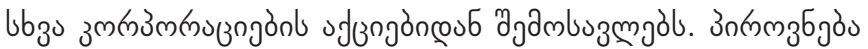

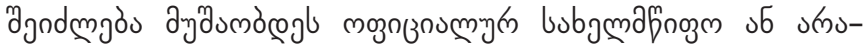

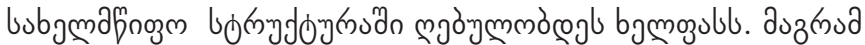

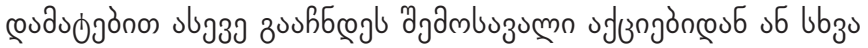
jmbुònçú; 


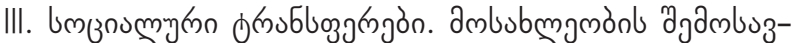

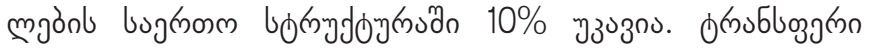
(ogrubb3. Transfert, moonobygnoce Transferre - zucououbs,

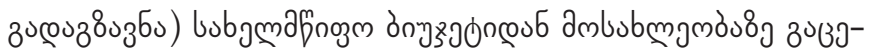

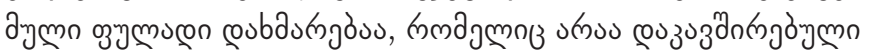

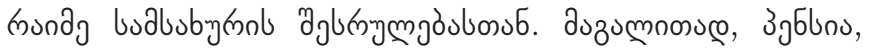

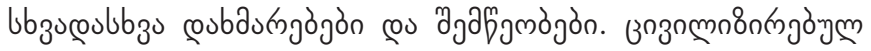

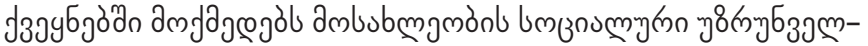

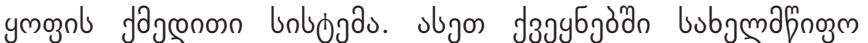

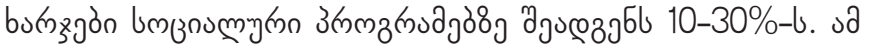

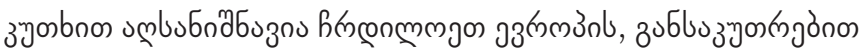

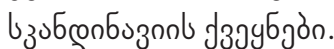

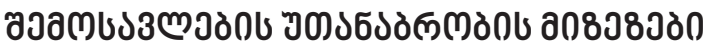

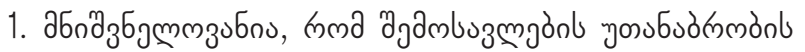

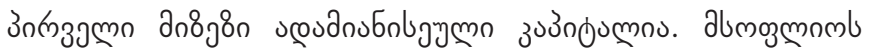

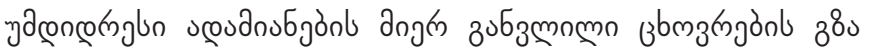

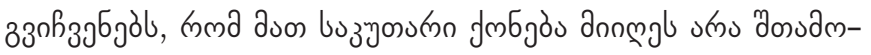

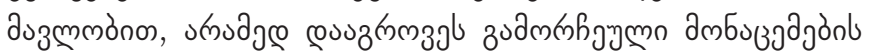
bugyd3aming.

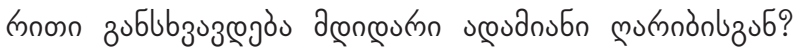

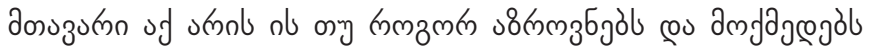
उпюмзुб

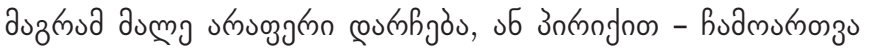

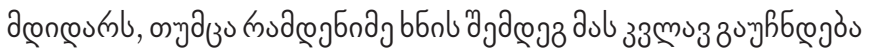
unacongorng.

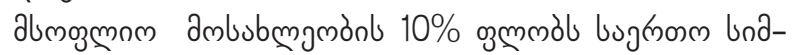

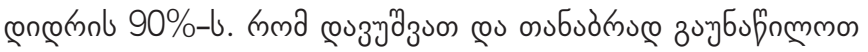
uмб

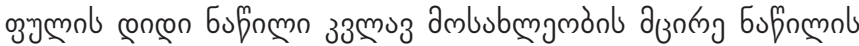

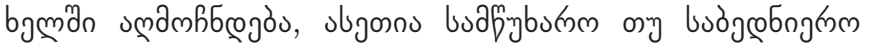
ingummós.

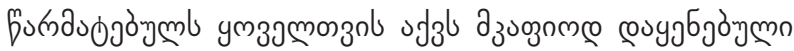

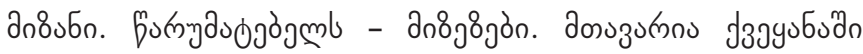

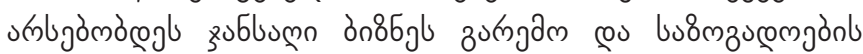

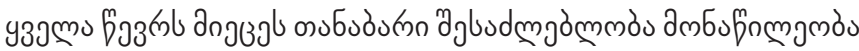

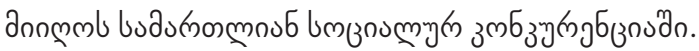

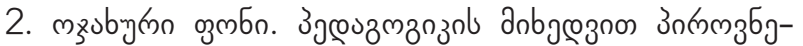

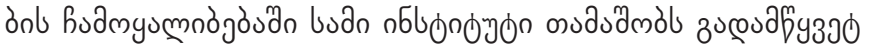

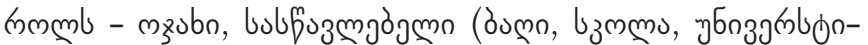

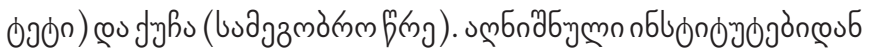

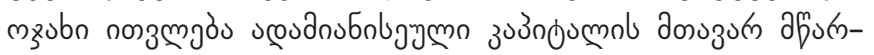

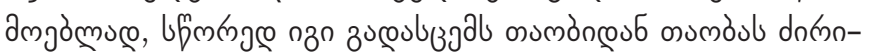

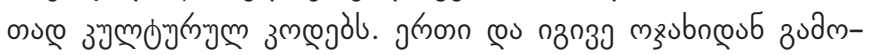

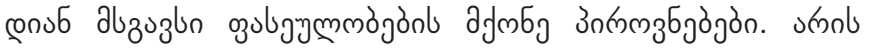

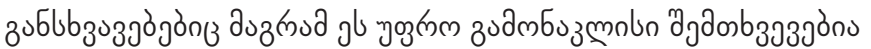
cos unu foplo (Bedianashvili, 2018).

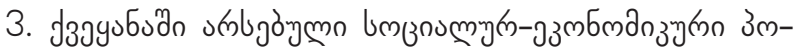

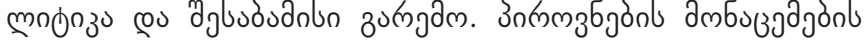

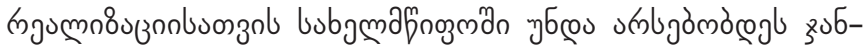

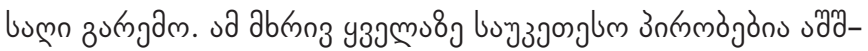

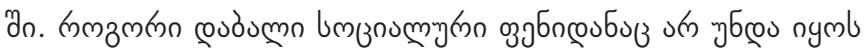

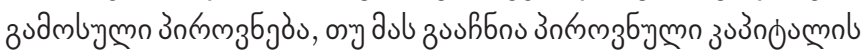

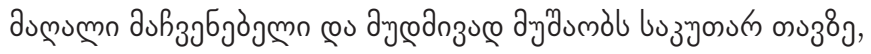

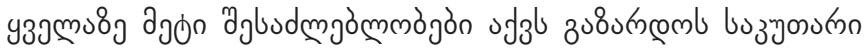

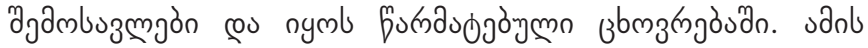

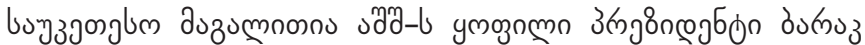

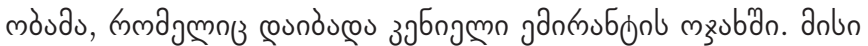

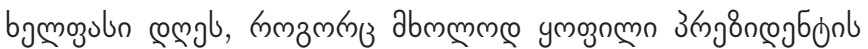
400 sonul communt aguesjob;

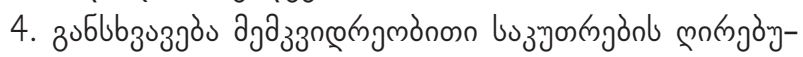
mgö

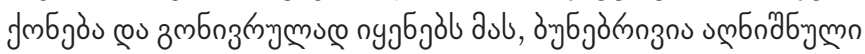

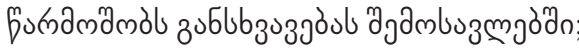

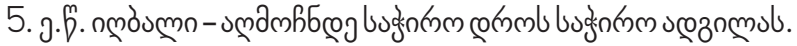

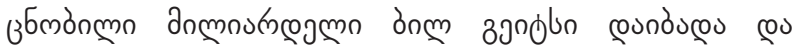

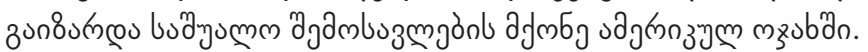

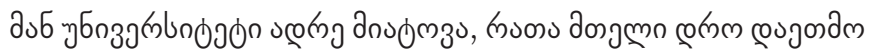
IT osfbmmmznjönbsonzol.

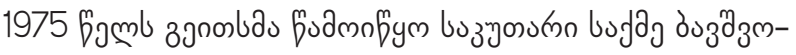

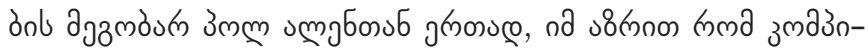

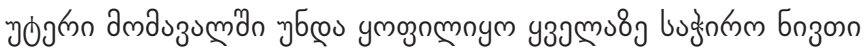

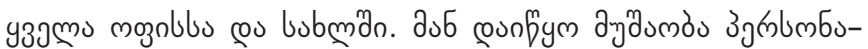

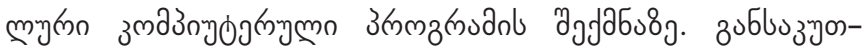

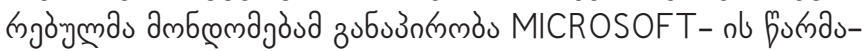

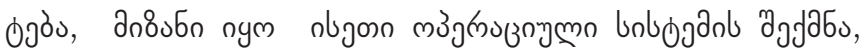

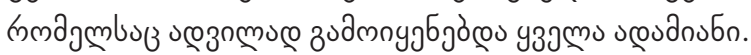

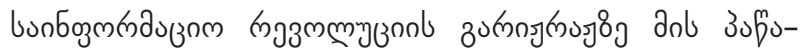

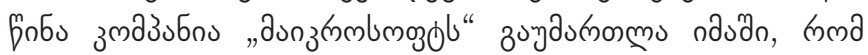

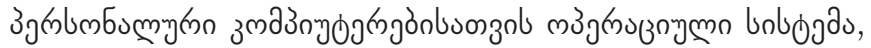

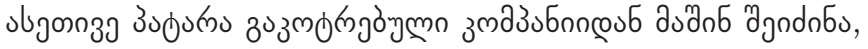

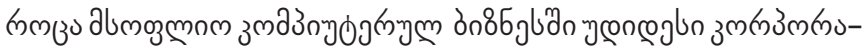

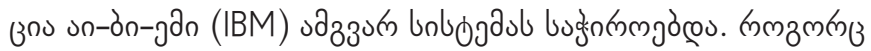

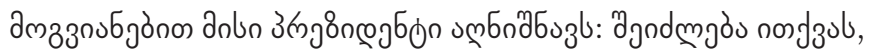

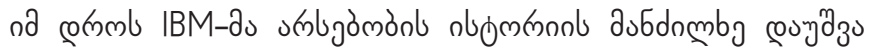

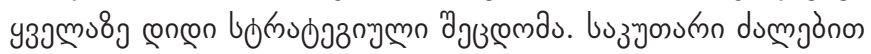
उח د

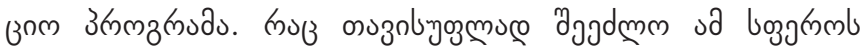

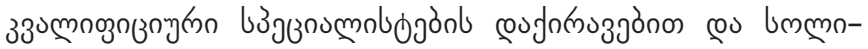

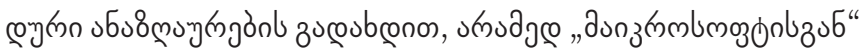

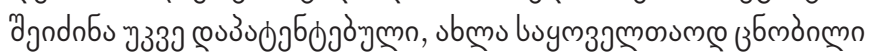
bumżgroznm bnboga MS-DOS.

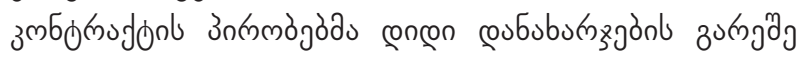

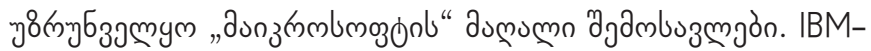

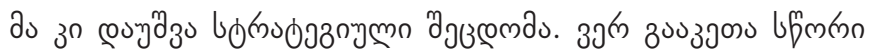

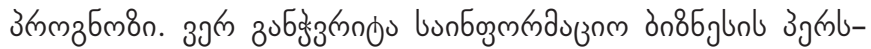

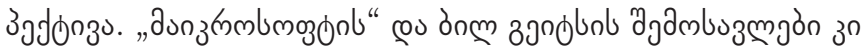

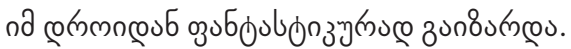




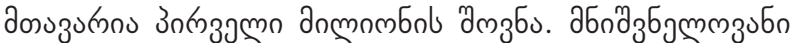

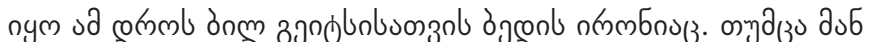

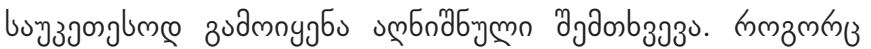

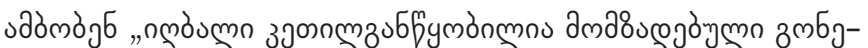
onb ugouanubjönbuonzol".

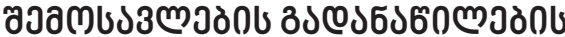

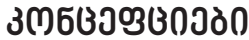

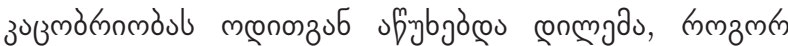

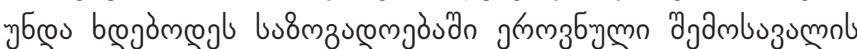

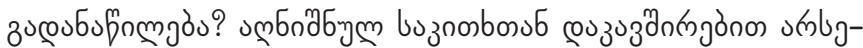

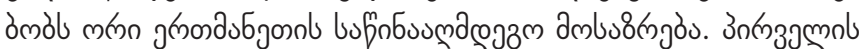

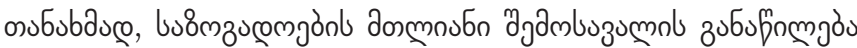

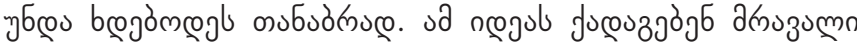

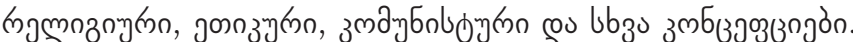

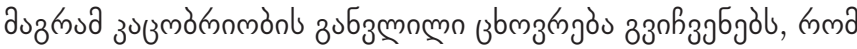

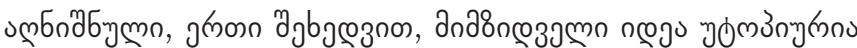

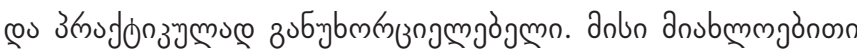

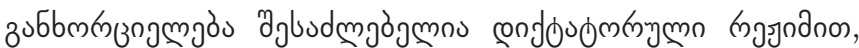

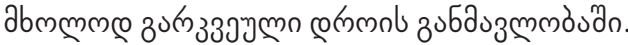

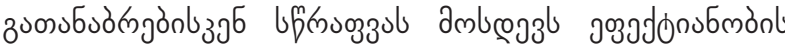

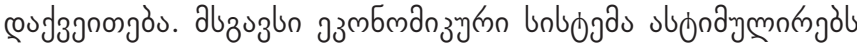

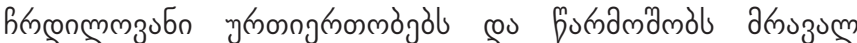

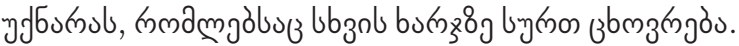

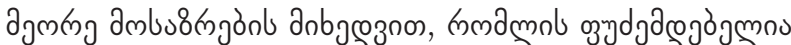

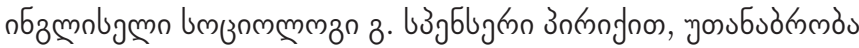

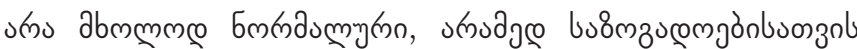

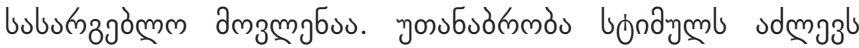

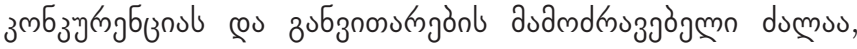

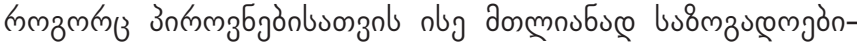

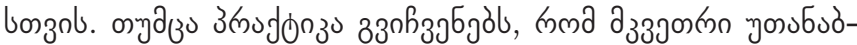

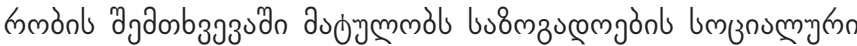

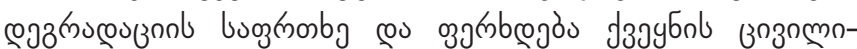

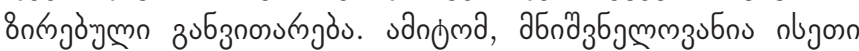

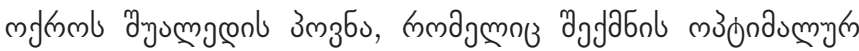

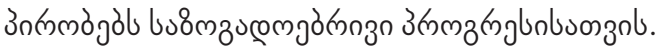

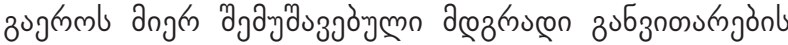

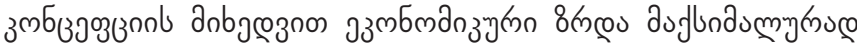

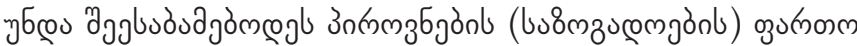

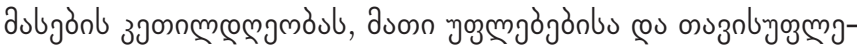

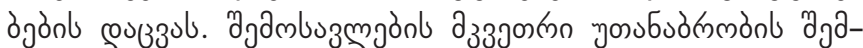

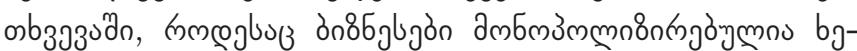

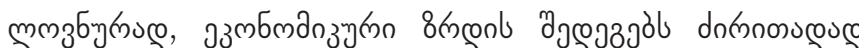

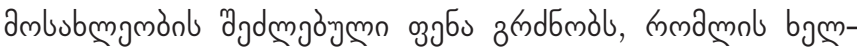

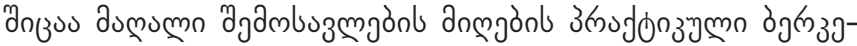

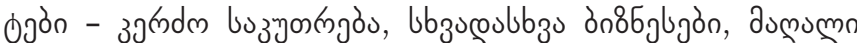

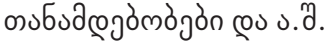

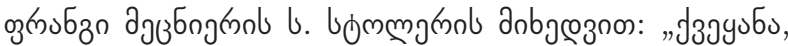

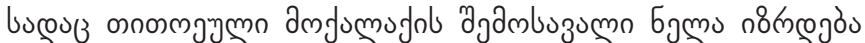

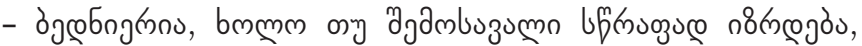

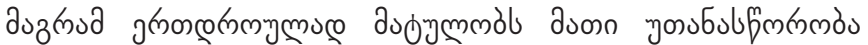

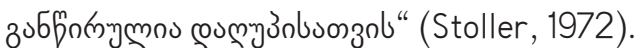

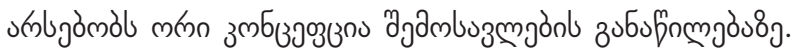

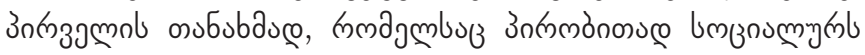

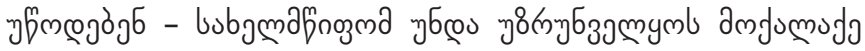

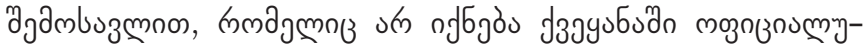

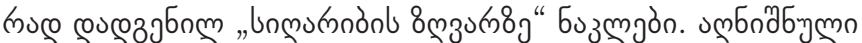
дnœ̧

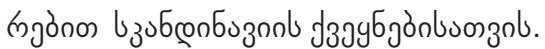

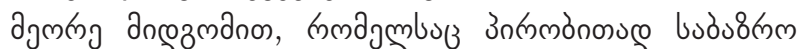

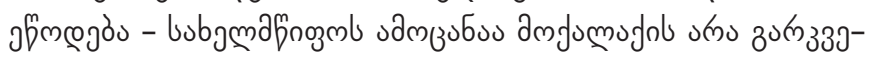
चmn zu

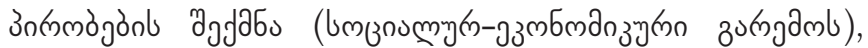

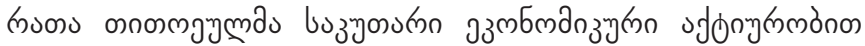

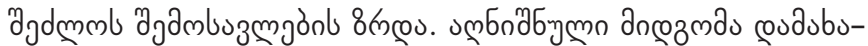

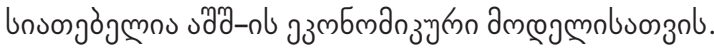

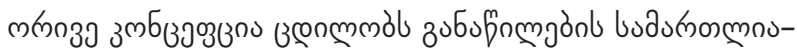

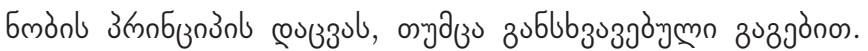

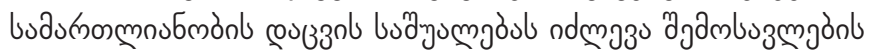

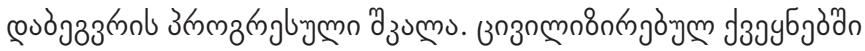

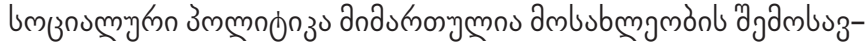

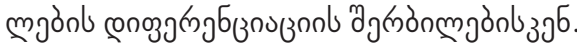

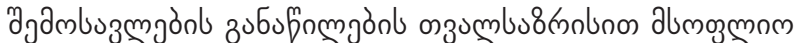

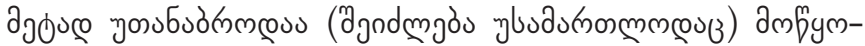

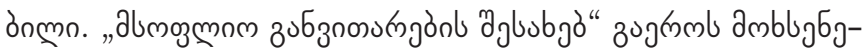

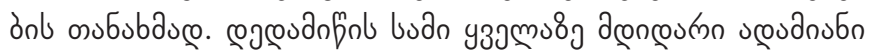

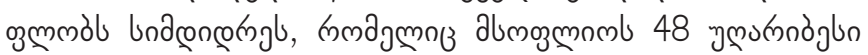

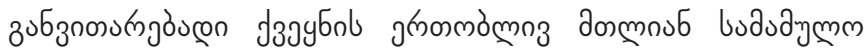

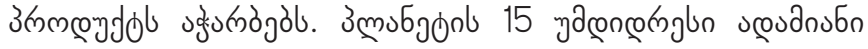

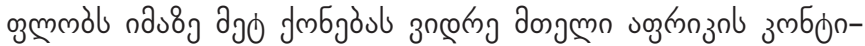

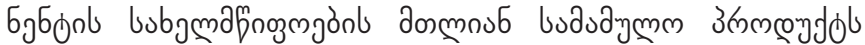

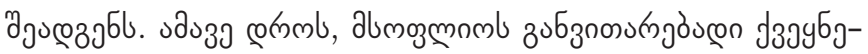

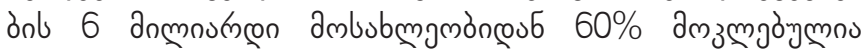

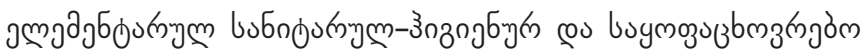

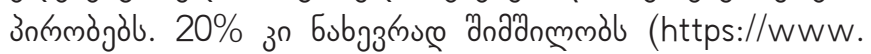
businessinsider.com/the-15-richest-people-in-africaranked-2020-9).

\section{@su336s}

ง

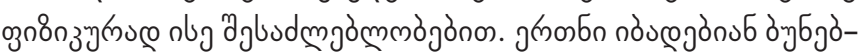

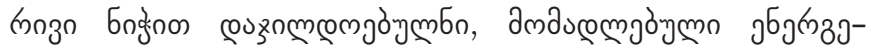

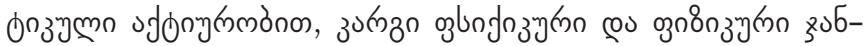

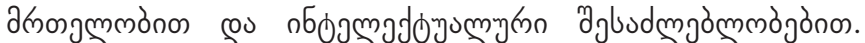

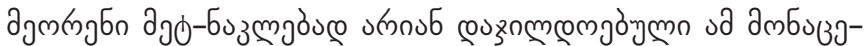

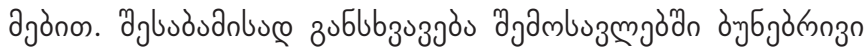




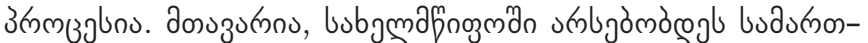

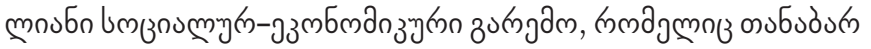

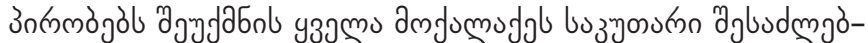

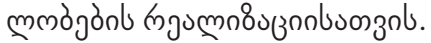

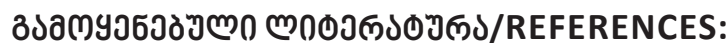

Abralava, A., (2014). Global-Innovative Problems of Economy and Business. Universal Publishing House, Tbilisi:612.

Avramova, E.M., Loginov D., (2002). Socio-economic adaptation: resources and opportunities. Journal ONS, 5:24-34.

Becker, G., (1983). Treatise of the Family, Oxf., 44

Bedianashvili, G., (2018). Culture as a factor of knowledge economics with paradigmatic changes in systemic institutional context. Globalaizatin and Business, 6:58-66.

Coleman, I., (1990), Foundations of Social Theory. Cambridge:42-54.

Country List Government Debt to GDP. Trading Economics (2019). https://tradingeconomics.com/country-list/governmentdebt-to-gdp

Global wage growth lowest since 2008, while women still earning 20 per cent less than men (2018).https://www.ilo.org/ global/about-the-ilo/mission-and-objectives/features/WCMS_650551/lang--en/index.htm

http://hdr.undp.org/sites/default/files/hdr2020.pdf

https://www.businessinsider.com/the-15-richest-people-in-africa-ranked-2020-9

Moskvin, L., (1999). New social measurements in the modern world, ONS, 1999, N3,

Papava, V., (2018), The Crisis of Economic Science and the Search for a New Paradigm, Globalaizatin and Business, 6:15-27.

Papava, V., Silagadze, A., (2019). How the Term the "Gross Domestic Product" should be Translated into Georgian. Globalization and Business, No. 7:203-204. (In Georgian)

Sartania, V., Tokmazishvili M., Maglakelidze Sh., (2004). Principles of Education Economics in the History of Public Opinion, Tbilisi, 391.

Stoller, L., (1972), Ravnovesie and йкономический рост. - M .: Progress, 440 p.

The Economic Significance of Natural Resources: Key Points for Reformers in Eastern Europe, Caucasus and Central Asia (2011). OECD. http://www.oecd.org/env/outreach/2011_AB_Economic\%20significance\%20of\%20NR\%20in\%20EECCA_ENG.pdf 